\title{
Models for optimally controlling varicella and herpes zoster by varicella vaccination: a comparative study
}

\author{
Monica Betta ${ }^{12}$, Alberto Landi ${ }^{2}$, Piero Manfredi ${ }^{3}$, Marco Laurino $^{4}$ \\ 1 IMT School for Advanced Studies, Piazza S. Francesco 19, 55100 Lucca, Italy \\ 2 Department of Information Engineering (DIE), University of Pisa, Via G. Caruso 16, 56122 Pisa, Italy \\ 3 Department of Economics and Management, University of Pisa, Via Cosimo Ridolfi 10, 56124 Pisa, Italy \\ 4 Institute of Clinical Physiology, National Research Council, Via Giuseppe Moruzzi 1, 56124 Pisa, Italy
}

\begin{abstract}
The introduction of mass vaccination against Varicella-Zoster-Virus (VZV) is being delayed in many European countries mainly because of the fear of a subsequent boom in natural herpes zoster (HZ) incidence in the first decades after the initiation of vaccination, caused by the expected decline in the protective effect of natural immunity boosting due to reduced virus circulation. Optimal control theory has proven to be a successful tool in understanding ways to curtail the spread of infectious diseases by devising the optimal disease intervention strategies. In this paper, we describe how a reduced 'toy' model can extract the essentials of the dynamics of the VZV transmission and reactivation in case of the study of optimal paths of varicella immunization programs. Results obtained using different optimization approaches are compared with the ones of a more realistic age-structured model. The reduced model shows some unreliable predictions in regards of model time scales about herpes zoster dynamic; nevertheless, it is able to reproduce the main qualitative dynamic of the more realistic model to the different optimization problems, while requiring a minimal number of parameters to be identified.
\end{abstract}

Keywords Models . Varicella . Herpes zoster . Optimal control . Vaccination

\section{Introduction}

Chickenpox and shingles are the two clinical manifestations of the same virus, the Varicella-Zoster-Virus (VZV) [1]. First exposure to VZV yields Varicella disease ('chickenpox'), a highly infectious disease, occurring especially in children [1]. After contracting varicella, people remain immune to such disease, but the virus remains latent and can reactivate at later ages, causing shingles in about $30 \%$ of those exposed to varicella.

Shingles, better known as herpes zoster (HZ), is a severe skin pathology that can cause serious morbidity, first of all post-herpetic neuralgia (PHN) [1].

Despite mechanisms underlying $\mathrm{HZ}$ pathogenesis remain still poorly clarified, a general consensus among the authors has been reached about the central role played by CMI decline with age in VZV reactivation [2,3].

Within this context, Hope-Simpson also introduced the exogenous boosting (EB) hypothesis, according to which CMI might be boosted by the re-exposure to VZV through further contacts with infective individuals.

Under this crucial assumption, the VZV circulation level represents in turn a critical parameter in modulating the mean VZV reactivation time.

Since 1970s, a vaccine for varicella has been available and mass childhood immunization programs against varicella infection were introduced in the USA (1995), Japan, and Australia. In Europe, the introduction of varicella immunization is much more limited and only a few countries follow full immunization programs [4].

The reason for this policy paralysis is largely due to the fear that the natural HZ may increase, as predicted by VZV models including the EB hypothesis and standard childhood programs, with a constant high vaccination coverage [515]. A straightforward question is whether alternative, time varying varicella-vaccination programs may improve performance in controlling both varicella and natural zoster [16]. The scenario is complex, because the vaccine strain itself may cause people to develop HZ [17]. Optimization algorithms can be considered in this complex task, namely control two epidemiological targets by a single control input, the varicella vaccination.

Despite the wide literature on optimal control applied to infection models $[18,19]$ only in reference [16], this problem was fully investigated, taking into account both the feasibility of the vaccination program and the mitigation of the natural zoster boom.

In reference [16], an age-structured model was considered, in order to obtain results robust enough for convincing the epidemiological community, since it provides a more realistic representation of the epidemiological problem. This model, though very rich in terms of epidemiological details, is also very demanding in terms of data, since its parameterization required both finely age-structured serological data documenting varicella infection, social contact data, and herpes zoster incidence data, which are available only for a few countries worldwide. Therefore, in order to help the health-related sector to assist public health decisions on infections and vaccination in countries missing such refined data, the key strategy is relying on much simpler models requiring a minimal number of parameters to be identified. In relation to this, the major, and very recent, example is represented by the modeling work of the large Ebola epidemics in West Africa in 2014-2015 where, given the lack of fundamental epidemiological knowledge, the 
early adopted models were necessarily oversimplified despite the complexity of the infection (see, e.g., [20, 21]). From an engineering point of view, this leads to an old standing question namely, whether a lower order model could provide acceptable qualitative results. It is the usual dilemma related to the search of an effective balance of the model complexity: low order models are often too simple to realistically simulate real system evolution, while on the other hand, the majority of high order models might suffer the disadvantage of being too demanding in terms of parameterization and control purposes. In this work, we make an effort to evaluate the selection of candidate reduced model that could simulate the main outstanding (within the international debate) epidemiological effects (namely the boom in natural herpes zoster and the slow increase of vaccine-related zoster following childhood varicella immunization) and at the same time could be sufficiently simple to avoid unsolvable parameterization issues preventing the solution of the optimal control problem. In our analyses, we therefore compare the results coming from our simpler epidemiological formulation with those of the realistic model adopted in reference [16], taken as the reference benchmark.

\section{Methods}

The age-structured and the low-dimensional reduced models

The fully age-structured model (ASM) for VZV of [16] describes the dynamics of VZV transmission and reactivation within a population that is stationary in terms of total size, with a constant number of births per year, and an agespecific mortality rate depending on the age groups. The model uses a set of 176 ordinary differential equations and considers 5year age groups, up to the age limit of 80 years. Mimicking the structure adopted in the most recent public health literature [5-9, 11, 12], it includes 11 epidemiological compartments and the flow diagram of the transition states for each age group is shown in panel a of Fig. 1.

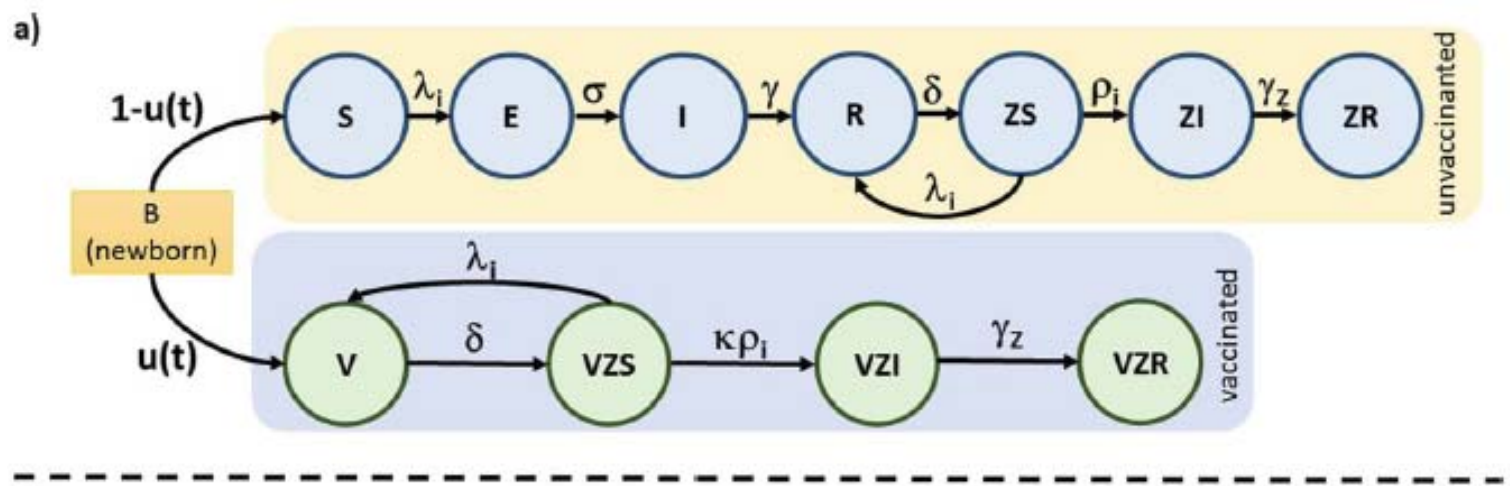

b)



Fig. 1 Flow diagrams for the realistic age-structured model (ASM) (a) and the simplified low dimensional reduced model (LRM) (b). Each box in the diagram represents a compartment while arrows report the transfer rates between model compartments

The ASM splits the population into two main arms of the scheme: unvaccinated individuals and vaccinated at birth against varicella by a vaccine supposed to be $100 \%$ effective and lifelong protecting.

In the panel a of Fig. 1, the transitions of individuals not immunized are shown: susceptible individuals (S) may contract varicella chickenpox from contacts with infective individuals (I) at an age depending on the force of infection (FOI) $\lambda_{i}(\mathrm{t})$. They move first to the exposed (E), than to the infective (I) state at constant rate $\sigma$, until they recover at constant rate $\gamma$. Recovered individuals (R) remain immune to chickenpox, but reduce CMI becoming susceptible to shingles (ZS) at rate $\delta$. ZS subjects may follow two different paths, either developing HZ at an age-specific rate $\rho \mathrm{i}$ becoming $\mathrm{HZ}$ cases $(\mathrm{ZI})$ or receiving a boosting of CMI, getting back to R class. ZI subjects recover at constant rate $\gamma_{z}$ becoming immune to shingles (ZR). Vaccinated individuals $(V)$ instead follow the lower arm of panel a of Fig. 1 with 
different transitions: they are fully immunized against chickenpox, but reduce vaccine-acquired CMI, becoming susceptible to HZ (VZS). VZS individuals may follow two different paths, either developing shingles (VZI) and then recovering from zoster disease (VZS), or receiving a boosting of CMI at rate $\lambda_{i}$, getting back to $V$ class.

The age-structured model is very complex, as it involves the insertion of age-dependent parameters, explained in detail in the supplementary materials of reference [16].

The aim of this paper is to present a simpler ordinary differential equation (ODE) model for VZV transmission and reactivation, mimicking the compartmental structure of the more realistic age-structured models used in reference [16]. This low dimensional reduced model (LRM) considers a stationary through identical birth and death rates $(\mu)$ and homogeneously mixing population with constant size $\mathrm{N}$, which is sub-divided into two subgroups, those not vaccinated and those vaccinated against chickenpox as in panel b Fig. 1, but removing the hypothesis of age-dependent groups. The model administers the vaccination to a time-varying fraction of newborn individuals in the hypothesis of a fully effective and lifelong protection against varicella virus. In the unvaccinated group, the acquisition of varicella follows the standard SIR course, where S(t), I(t), and R(t) denote numbers of individuals who are susceptible, infectious, and recovered to varicella at time t. In particular, all unvaccinated individuals are susceptible to varicella (S), which they acquire at a time-dependent rate $\lambda=\lambda(\mathrm{t})$ becoming infectious (I). Infectious individuals are also infective and recover at constant rate $\gamma=1 / D$, where $D$, is the average duration of varicella infection, here kept fixed to 1 week. Recovered individuals $(\mathrm{R})$ are permanently immune to varicella but become susceptible to zoster (ZS) at constant rate $\delta$ mimicking the decline in CMI over time. ZS individuals either develop HZ at a constant rate $\rho$, becoming active zoster cases (ZI), or receive an exogenous boosting (at constant rate $\lambda \mathrm{z}$ ) of CMI through reexposition to VZS, getting thus back to the ZS class. Active zoster cases recover at constant rate $\gamma z=1 / \mathrm{D} z$ becoming immune to zoster (ZR). The varicella force of infection is defined as $\lambda=\beta \mathrm{I} / \mathrm{N}$.

$\mathrm{N}$ by assuming that the contribution from zoster infective individuals is negligible, as documented in the literature [6]. Exogenous boosting $\lambda z$ is assumed to occur at a force of boosting identical to the force of infection $\lambda$. As the agestructured model, the vaccinated individuals are fully protected against varicella infection but they reduce CMI acquired through the vaccine at the same decay rate $\delta$ of natural CMI becoming susceptible to HZ (VZS) which they acquire at a reduced rate $\rho \mathrm{v}=\mathrm{k} \rho,(0<\mathrm{k}<1)$, entering VZI class. Boosting of vaccinated individuals is neglected in this simplified model. Mathematical equations for the LRM model are in Table 1. Definitions, dimensional units, assignments, and related literature sources for all parameters of the VZV model usedin the main text, and for all relevant control parameters are summarized in Table 2. In particular, the varicella transmission rate $\beta$ is computed by assigning the basic reproduction number $R_{0}$ and then computing $\beta$ as $\beta=(\mu+\gamma) R_{0}$.
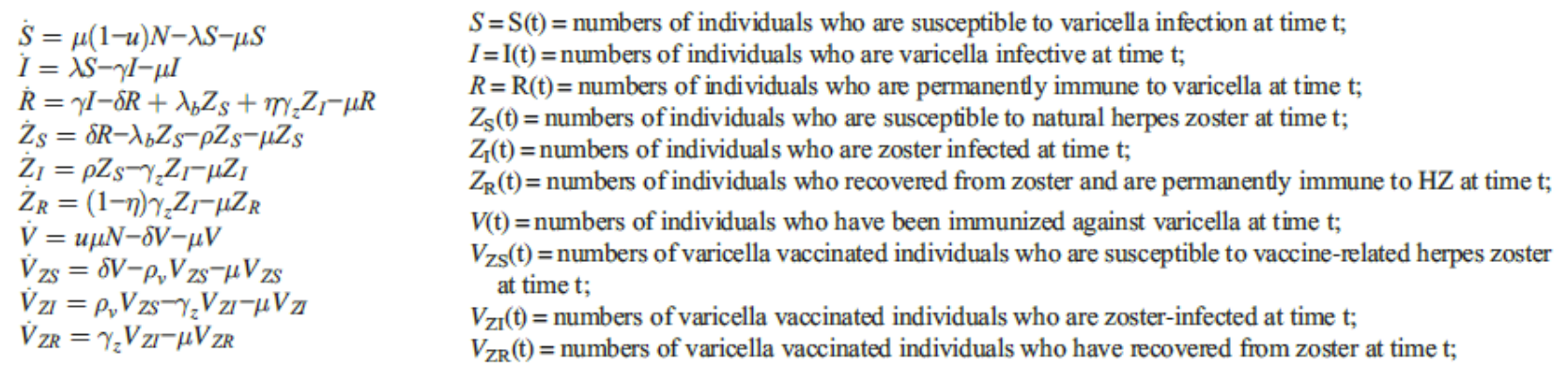

Table 1 Equations for the low-dimensional reduced model (LRM) reported in the main text with full description of variables 


\begin{tabular}{lllll}
\hline Parameters & Unit & Adopted values & Baseline & Source \\
\hline$T=$ duration of control horizon & Year & {$[30,50,100]$} & - & Free simulation parameter \\
$c_{Z}=$ relative cost of a zoster case with respect to a varicella case & - & {$[1,10,30]$} & - & Free simulation parameter \\
$r=$ discount rate & Year $^{-1}$ & Fixed & 0.03 & From [22] \\
$\mu=$ death rate (=1/life expectancy) & Year $^{-1}$ & Fixed & $1 / 75$ & - \\
$R_{0}=$ basic reproduction number of varicella & - & Fixed & 6 & From [23, 24] \\
$\gamma=$ varicella recovery rate & Year $^{-1}$ & Fixed & 52.1 & From $[1]$ \\
$\beta=$ varicella transmission rate & Year $^{-1}$ & Fixed & 312.1 & $\beta=(\mu+\gamma) R_{0}$ \\
$\delta=$ duration of CMI protection & Year $^{-1}$ & Fixed & 0.05 & From $[8,13]$ \\
$z=$ boosting fraction & $\%$ & Fixed & 100 & From $[8,13]$ \\
$\rho=$ hazard rate of natural zoster & Year $^{-1}$ & Fixed & 0.0667 & From $[8,13]$ \\
$\gamma_{Z}=$ zoster recovery rate & Year $^{-1}$ & Fixed & 24.3 & \\
$(=1 /$ average duration of zoster episodes) & & & 0.125 & From $[17]$ \\
$k=$ reduction factor of zoster hazard for varicella vaccinated individuals & - & Fixed & 4 & - \\
$G=$ weight of the augmenting component in augmented cost functional & - & Fixed & & 4 \\
\hline
\end{tabular}

Table 2 Table with definitions, dimensional units, numerical ranges, and related literature sources for all parameters of the optimal control problem and the LRM

\section{The optimal control problem and its formulations}

\section{Full optimal control problem}

We define as full optimal control problem the formulation that seeks the time path $u(t)$ of yearly routine varicella vaccination that minimizes total discounted costs arising from varicella and HZ (both natural and vaccine-related), according to the functional:

$$
C_{[0, T]}=\int_{0}^{T} e^{-r t}\left(\beta I(t) S(t)+c_{Z} \rho Z S(t)+c_{Z} \rho_{V} V Z S(t)\right) d t
$$

where $r$ is the discount rate, which mitigates costs for long horizons (case of $r>0$ ), [0,T] is the planning horizon, $\beta \mathrm{l}(\mathrm{t}) \mathrm{S}(\mathrm{t}), \rho Z \mathrm{~S}(\mathrm{t}), \rho \vee V Z S(\mathrm{t})$, respectively are the cases of varicella, natural $\mathrm{HZ}$, and vaccine-related $\mathrm{HZ}$ at time $\mathrm{t}$, and $\mathrm{cz}$ represents the cost of a unit zoster case relative to that of a varicella case. In the cost functional (1), the control action $\mathrm{u}=\mathrm{u}(\mathrm{t})$ is embedded implicitly, through the states' dynamics. The approach underlying eq. (1) is that of a 'social planning'problem where a social planner, namely the public health system, seeks to minimize the overall costs arising from the various items of the disease burden.

Note that the cost function (1) is the same as that proposed in reference [16], but referred to the simplified model without age studied in this paper. In simple words, in both problems, we aim at minimizing costs arising from total cases of varicella and zoster diseases.

The control function $u=u(t)$ is allowed to vary yearly, because we believe that 1 year is the minimal updating time affordable in a real public health framework. Nonetheless, for completeness, we have also considered shorter updating times (6 and 3 months, respectively) without finding noteworthy differences (see appendix 1). In (1), we can highlight two critical parameters, namely the length of the planning horizon $\mathrm{T}$, and the relative $\mathrm{HZ}$ cost $\mathrm{Cz}$. In this work for the planning horizon $(\mathrm{T})$, we considered different scenarios, ranging from medium-term policies ( $\mathrm{T}=30 \mathrm{yrs})$ up to $\mathrm{T}=100 \mathrm{yrs}$. For the relative ZI cost, considering the large variability of literature estimates $[11,25,26]$ and the uncertainty surrounding critical aspects, e.g., the reduction in quality of life due to PHN, we decided to take $\mathrm{Cz}$ as a free simulation parameter ranging between 1 and 30. The discount rate is fixed at the baseline value $r=0.03 / \mathrm{yrs}$, as commonly used in economic evaluations of immunization programs [7, 15].

These assignments are summarized in Table 2.

In simple control models with a linear cost function, Pontryagin's maximum principle [27] yields the classic bang-bang solution, switching between the bounds of the control set. The problem at hand has a realistic constraint, i.e., the constancy of the optimal vaccination coverage within each year. Furthermore, many factors contribute to modify the classic solution, because the problem has (i) nonlinear dynamics, (ii) a single input for controlling two outputs, (iii) uncertainty surrounding the relative costs of zoster, and (iv) different durations of possible control horizon.

\section{Logistic optimal control problem}

Fully optimal vaccination control functions can have a complicate temporal behavior (e.g., oscillations), resulting practically unfeasible, e.g., due to community costs, ethical problems, etc. We therefore also considered restricted functional forms of the control function, i.e., logistic, aimed to represent smooth feasible immunization programs: 


$$
u_{l}(t)=\frac{a}{1+b \cdot e^{-c t}}+d t>0
$$

This choice of a logistic control function is motivated allowing an early phase with boosting opportunities, where the VZV circulation remains limited, to mitigate the natural HZ boom following in case of a hard initial vaccination policy.

\section{Free optimization problem with augmented cost functional}

One might expect that for very long planning horizons (say, in excess of 70-100 years), varicella elimination may be optimal regardless of the fact that it caused the natural HZ boom. The intuition underlying the sentence is simply that for very large horizons, the zoster wave, which lasts a few decades only, is forgotten and therefore the dominating cost items are those due to varicella and vaccine-related zoster cases (which have a lower risk compared to natural zoster). Therefore, it is not surprising that the optimal strategy becomes varicella elimination regardless the magnitude of the relative cost of $\mathrm{HZ}$. Therefore, we considered the following augmented cost function:

$$
\begin{aligned}
A C(T)= & \int_{0}^{T} e^{-r t}\left(\beta I(t) S(t)+c_{Z} \rho Z S(t)+c_{Z} \rho_{V} V Z S(t)\right) d t \\
& +G \int_{0}^{T} e^{-r t} c_{z} \max \left(0, \rho Z S(t)-\rho Z S_{u=0}(t)\right) d t
\end{aligned}
$$

Formulation (3) adds to the baseline cost function (1) a term which is strictly positive only during the epoch, of duration $\mathrm{T}_{\mathrm{A}}$, in which the number of natural $\mathrm{HZ}$ cases lies above its prevaccination level $(\mathrm{u}=0)$. $\mathrm{G}>0$ is a free parameter tuning the weight of the augmenting component with respect to the baseline one. The purpose of considering the augmented functional is that of including an extra penalty for policy makers who deliberately introduce varicella vaccination knowing that it will worsen, at least temporary, the existing situation.

\section{Solution of the optimal control problem}

The optimal program is performed to minimize the scalar cost (1) or (3) over the whole horizon. The control function, i.e., the vaccination coverage $\mathrm{u}(\mathrm{t})$, is allowed to vary between $0 \%$ (no vaccination) and a maximum feasible coverage of $90 \%$, i.e., exceeding the critical coverage $p_{c}=1-1 / R_{0}$, to allow greater flexibility. The optimality system is solved numerically and the solution of (1) is obtained, at each sampling time, using Matlab nonlinear constrained minimization routine fmincon [28]. To cope with local minima problems, a pool of 15 sets of initial conditions has been used as different starting points for the optimization algorithm. The first five initial conditions have been generated assuming constant vaccination coverages $(10 \%, 30 \%, 50 \%, 70 \%, 90 \%$, respectively) overall the time horizon, while in the remaining ten cases, the initial values of the optimization parameters have been randomly chosen. The optimal coverage was eventually chosen as the one associated to the minimum value of the cost function among those obtained for the whole pool of initial conditions. In the case of the logistic control problem, optimization is carried out with respect to the parameters $(a, b, c, d)$ of the logistic curve (2). Details about the optimization range for the parameters of the logistic control function (2) are reported in the table below (Table 3).

\begin{tabular}{lllll} 
& $a$ & $b$ & $c$ & $d$ \\
\hline Lower bound & 0 & 1 & 0.01 & 0 \\
Upper bound & 0.9 & $+\infty$ & 1 & 0.9 \\
\hline
\end{tabular}

Table 3 Optimization range for the parameters ( $a, b, c, d)$ of the logistic control function (formula (2) of main text)

\section{Results}

\section{The full optimal control problem}

We based the reported results on the evolutions of the models with the baseline configuration for epidemiological parameters (see Table 2), by letting the control parameters to vary. Figure 2 reports a sample of our results for a time horizon of 50 years and different levels of relative costs $\mathrm{Cz}$ of an $\mathrm{HZ}$ case, including both the optimal control function as 
well as of the resulting trajectories of the controlled system. Panel a of Fig. 2 reports the optimal time path $u(t)$ of vaccine uptake.

(a)
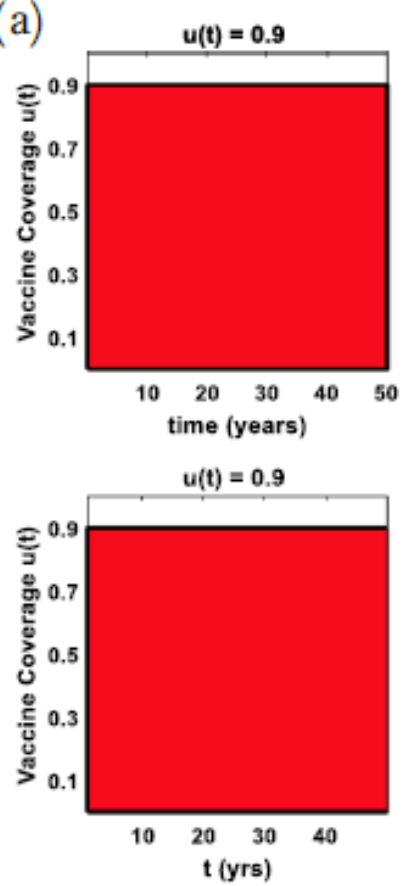

(b)


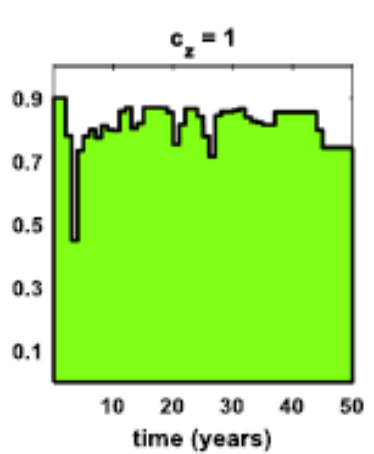

$c_{x}=1$



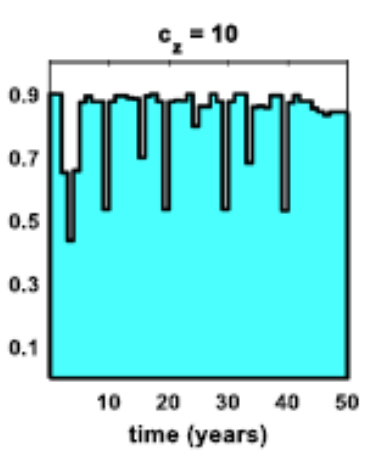

$c_{x}=10$

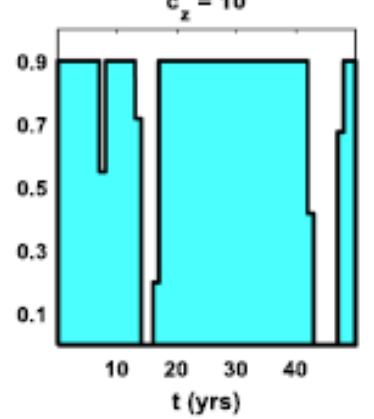

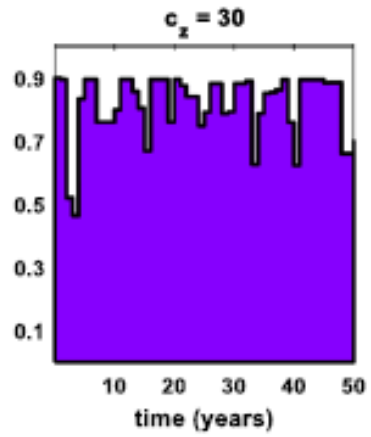

$c_{x}=30$

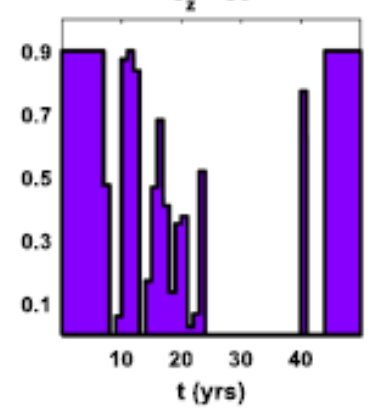

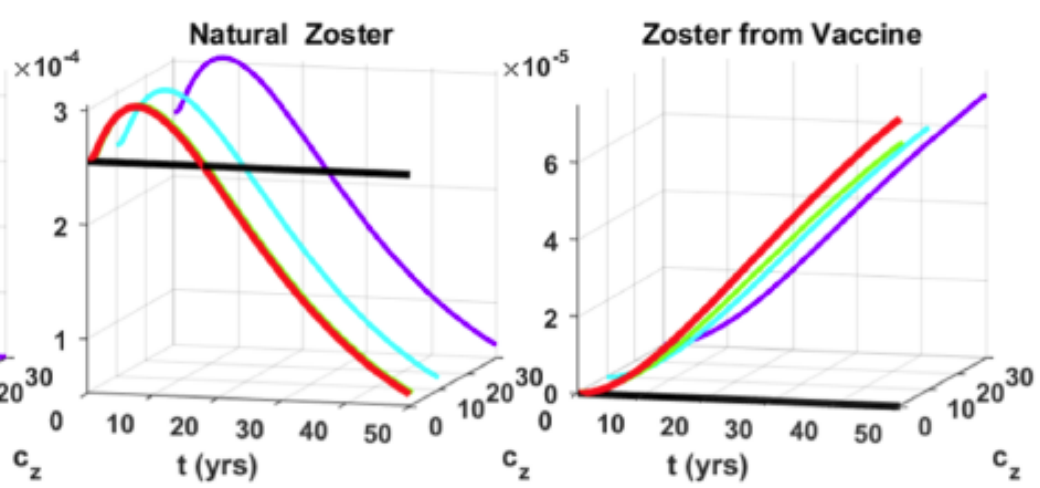

Natural Zoster
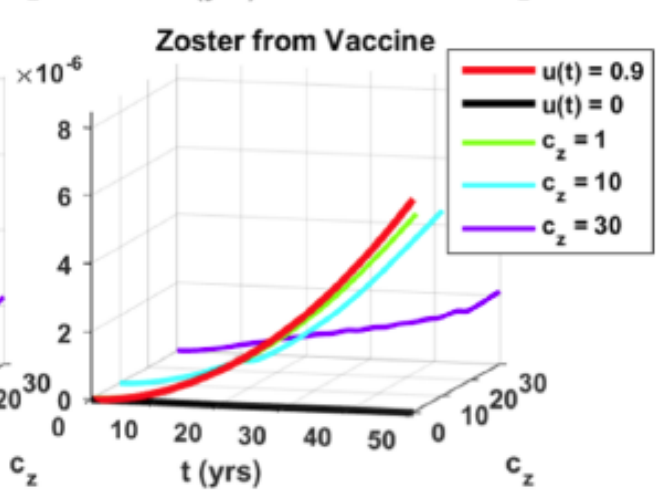

Fig. 2 The results for full optimal control problem.

a Solutions of the full optimal control problem (temporal trend of the optimal vaccination coverage $u(t))$ using, respectively, the realistic age-structured model (lower row) and the simplified model (upper row) for a 50-year planning horizon.

$\boldsymbol{b}$ Temporal trends of varicella cases (left), natural Zoster cases (middle), and vaccine-related Zoster cases (right) expressed as fraction of the total population, correspondent to the optimal temporal strategies of vaccine coverage showed in a for the realistic age-structured model (lower row) and the simplified model (upper row). Red and black curves represent the limit cases of a constant vaccine uptake set at the maximal level allowed $(u=90 \%)$ and without any immunization, respectively 
The upper row of panel a of Fig. 2 shows the results in the case of LRM while the lower row of panel a shows the results for the ASM. The corresponding temporal trends of varicella, natural HZ cases, and vaccine-related $\mathrm{HZ}$ cases are shown in panel b of Fig. 2 (upper and lower rows for the LRM and the ASM, respectively). The temporal horizon was chosen in order to compare the results of LRM with the ones of ASM in reference [16]. The effects of different lengths of the temporal horizon are reported in Fig. 3. For comparison purposes, we deliberately considered different horizons for the LRM and ASM models.

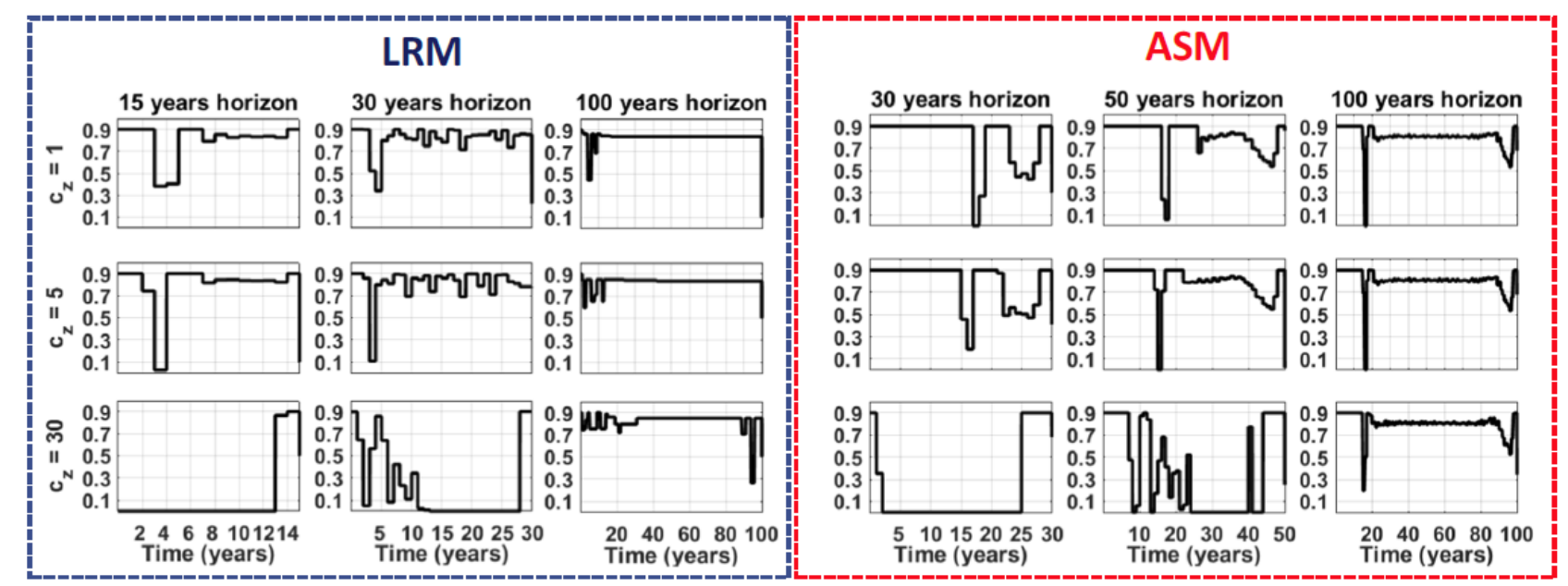

Fig. 3 Optimal vaccination programs for three different levels of relative cost of HZ case and three different control horizons are reported for the LRM and ASM model, on the right and on the left, respectively. The comparison between the two models is proposed over different horizon lengths, considering that the inclusion of age-structure brings a longer duration of the natural herpes zoster wave.

\section{Logistic optimal control problem}

Solutions of the logistic control problem for a planning horizon of 15 years are reported in Fig. 4. In particular, for a direct comparison, the right panel of Fig. 4 shows the optimal logistic paths of vaccine uptake for the LRM, with relative $\mathrm{HZ}$ ranging from 1 to 30, while the left panel reports the corresponding logistic optimal strategies for the ASM.
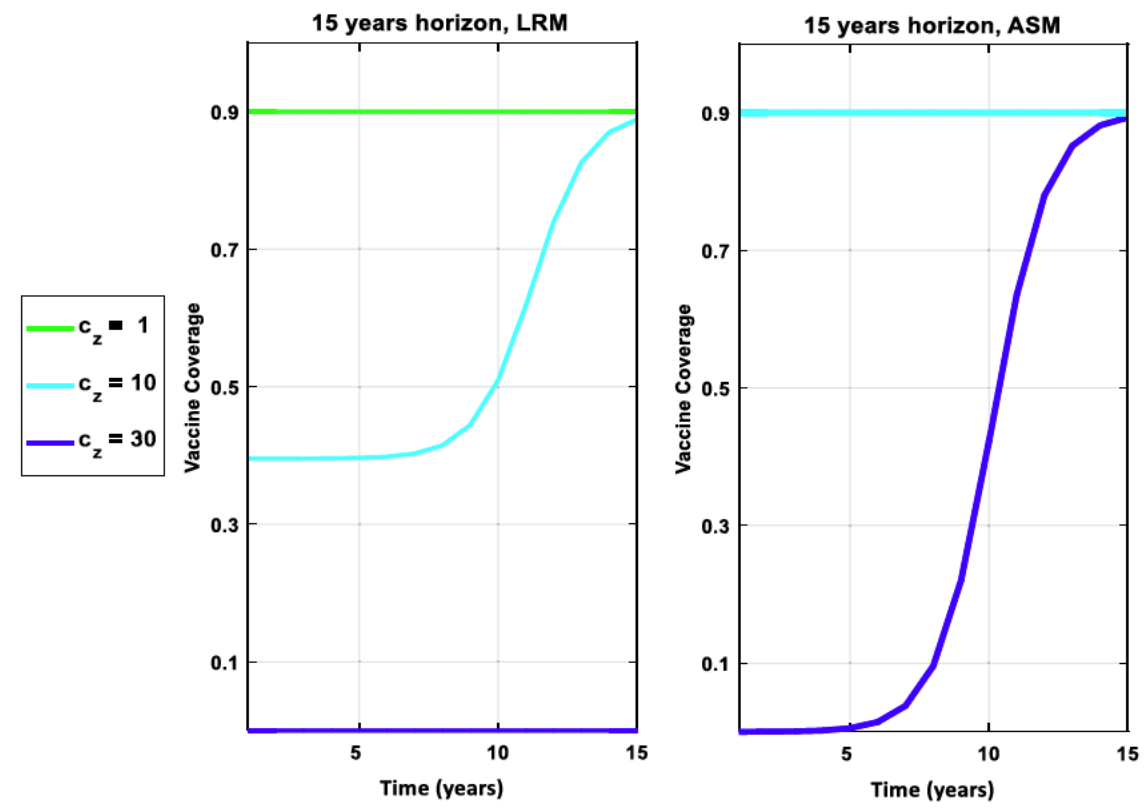

Fig. 4 Results for logistic optimal control problem. Optimal path of vaccine uptake, considering a logistically increasing coverage function for the realistic age structured model (a) and the simplified model (b). Here, the planning horizon is set to 15 years. 
Free optimization with augmented cost index

We report the results related to the free optimization with the augmented cost index only for long planning horizon $(T=$ 100) of optimization and the value of $\mathrm{HZ}$ cost set at 10, in order to compare the results with [16]. Figure 5 shows the results for both models and reports the corresponding trends for cases of (i) absence of any immunization $(\mathrm{u}(\mathrm{t})=0$, green lines), (ii) under the elimination program with constant vaccine uptake set at the maximum threshold $(\mathrm{u}(\mathrm{t})=0.9$, red lines), and (iii) the optimal vaccination coverage resulting from the minimization of augmented cost index (3) (uopt(t), black lines).
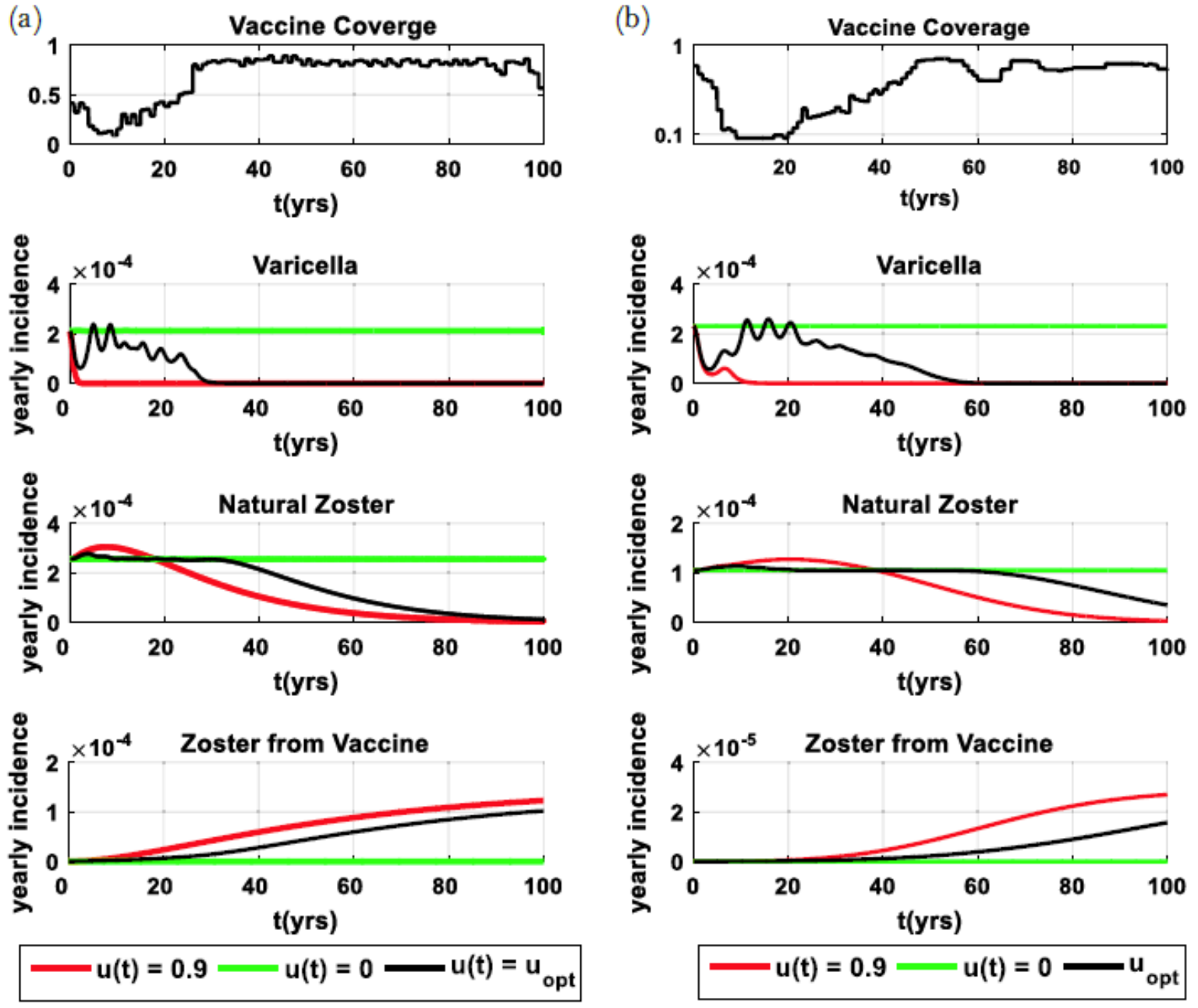

Fig. 5 Results obtained with free (a) (b) optimization with augmented cost index. Optimal vaccination strategy cases under the augmented cost index, and the corresponding temporal trends of varicella, natural, and vaccine related HZ for the simplified model at left panels (a) and the realistic age structured model at right panels (b), considering for both of them a 100-year planning horizon.

\section{Discussion}

The full optimal control problem

Figure 2 compares the results of the two models over the same control horizon of 50 years, to illustrate the characteristic dynamics of the optimal control functions and the dynamics of the resulting state variables of the controlled system. Despite the complex resulting shapes of optimal programs, we can distinguish some clear trends, common for the two models, considering the varicella vaccination strategies obtained over a wider set of control horizon. Figure 3 shows that the main features predicted by the ASM model are maintained by the LRM model: (a) when the relative cost of zoster is low and the horizon is short the optimal control program is to vaccinate against varicella at high coverage during essentially the entire planning horizon; (b) when the relative cost of $\mathrm{Hz}$ case $\mathrm{Cz}$ is high and the horizon is short, the optimal control program is (essentially) never vaccinate against varicella to avoid the cost of the herpes zoster wave; 
(c) for long horizons, the optimal control program is always vaccinate against varicella at high coverage irrespective of the cost of herpes zoster. Note that in Fig. 3, we compare the two models over different lengths of the control horizon. This was motivated by the fact that the inclusion of age-structure brings a longer duration of the natural herpes zoster wave, so that comparing the two models on the same horizons would be misleading. At high constant coverages (without optimal control), about the $80 \%$, the duration of the zoster wave is about 40 and 20 years for the ASM and LRM, respectively [13]. Note that the optimal programs show some irregularities due to the attempt, from time to time, depending on the parameters of the problem, to either reduce or increase the vaccine uptake in order to contain the costs arising from the two main items of the cost function, those due to varicella and herpes zoster disease, respectively.

\section{Logistic optimal control problem}

The complex resulting shapes of the full optimal control problem reported in Fig. 3 make them unfeasible for a real application from a practical public health viewpoint, as they often predict large oscillations in vaccine uptake and over a long-range horizon. For these reasons, we decided to restrict the functional forms of the control function to a logistic one. In this way, we aimed to obtain a smooth and feasible immunization program relevant in a short time horizon. In many cases, the optimal logistic solution might be trivial, e.g., selected in the flat initial or final portions of the curve, and therefore not differing from a constant vaccine uptake curve. What we are therefore seeking are non-trivial logistic coverage curves, i.e., characterized by a wide variation between its two asymptotes, allowing a large improvement in performance compared to constant vaccine uptake programs.

In these terms, the most interesting results appear for short-term planning horizons, that is for levels comparable with the time scale of the natural zoster boom (at about $T=20$ for LSM and about $T=40$ for ASM). Here, we report for a direct comparison the results obtained for a planning horizon of 15 years, value below the estimated minimum extension of the HZ boom for both models. In the case of logistic solution, the results are very sharp for both models (Fig. 4). The panel on the right of Fig. 4 shows the optimal logistic paths of vaccine uptake for the LRM, with different $\mathrm{HZ}$ costs ranging from 1 to 30 . At very low relative costs $\left(\mathrm{Cz}_{2}=1\right)$ of $\mathrm{HZ}$, the optimal logistic policy is a trivial one predicting varicella elimination by a constant vaccine uptake program $(\mathrm{u}(\mathrm{t})=0.9)$. Increasing $\mathrm{HZ}$ costs, the optimal program becomes a non-trivial logistic program with a gradual reduction of vaccine uptake as a function of increasing of $\mathrm{HZ}$ cost. For example, the optimal logistic program for $\mathrm{Cz}_{\mathrm{z}}=10$ requires to initiate vaccination at a high medium level uptake $(\mathrm{u}(\mathrm{t})=0.4)$ in the first 10 years, and then to progressively increase coverage at full levels $(\mathrm{u})(\mathrm{t})=0.9)$ in the subsequent 10 years. At high relative $\operatorname{cost} \mathrm{Cz}=30$, optimal solution becomes trivial again, suggesting to keep the vaccine uptake constantly equal to zero during all the planning horizon. The correspondent logistic optimal strategies for ASM are shown in the left panel of Fig. 4. Like the simplified model, when $\mathrm{Cz}_{\mathrm{z}}=1 \mathrm{or} \mathrm{Cz}=10$, the optimal logistic path is a trivial one and the vaccination uptake is set to the constant maximal level (uı(t) $=0.9)$. Also, for ASM, increasing $\mathrm{HZ}$ costs, the optimal program becomes a non-trivial logistic one with a progressive reduction of vaccination. Differently to LRM with the same relative HZ cost, the vaccine uptake is maintained higher than LRM in particular during the end of the logistic program. Considering the same HZ cost of previous example, in the case of ASM, the initial vaccination is set to an uptake level $(\mathrm{ul}(\mathrm{t})=0.76)$ and the logistic rising starts after the first 10 years similar to LRM. Nevertheless, the final vaccine uptake is set at lower level $(\mathrm{ul}(\mathrm{t})=0.87)$ than LRM, ensuring high but subcritical levels of varicella control, in order to allow some boosting to be maintained. At high relative $\mathrm{HZ}$ cost $(\mathrm{C} z=$ $30)$, the optimal logistic program becomes again a trivial one, but with very low level of vaccine uptake $(\mathrm{U}(\mathrm{t})=0.03)$.

The observed differences between LRM and ASM could be mainly due to the effect of boosting that is shorter in LRM than ASM, and the lower levels of vaccine uptake obtained for ASM result to maintain a high level of boosting for long time.

\section{Free optimization with augmented cost index}

Interestingly, the main result is that the optimal vaccine coverage obtained for both models are very similar and they approximate a logistic curve. As regards the LRM, except the initial transient, the optimal vaccine uptake starts at a low level (about 10\%); then, the vaccination increases in about 20 years to reach its maximum level (a range nearly 90\%), after the dynamic remains quite stable and with low-amplitude fluctuations until the end of optimization horizon. As within 30 years, the varicella is fully eradicated but simultaneously the vaccine-related $\mathrm{HZ}$ starts its progressive growth. The optimal program for ASM is capable to avoid any boom of natural HZ exists (Fig. 5b, bottom-centre box, black curve). Disregarding transient initial effects, this program must depart from very low coverage levels (in the range of $10 \%$ ), and then increase vaccine uptake slowly, reaching elimination levels in a few decades (within about 60 years). The optimal dynamic obtained for ASM is very similar to the one of LRM; the main differences are the slower increase of vaccine coverage and the presence of smooth oscillations for ASM. The lower growth rate of vaccine coverage of ASM is probably due to the long-term boosting of Natural Zoster with respect to LRM.

Obviously, in the case of ASM, the full mitigation of the zoster boom unavoidably pays the price of delaying a large part of natural zoster cases into the future, rather than fully avoiding them (Fig. 5b, bottom-center box, red curve), as expected given that the only control tool is the varicella vaccine. However, it has the advantage of keeping lower the incidence of vaccine-related $\mathrm{HZ}$ with respect to the constant maximum vaccine coverage. In addition, the vaccinerelated HZ resultant from ASM is nearly one order of magnitude lower than in LRM. Also in the case of the free 
optimization with augmented cost index, the main difference of dynamical behavior between the two models ASM and LRM is related to the different duration of boosting effect of Natural Zoster.

\section{Conclusion}

In Europe, the introduction of childhood varicella immunization is strongly debated, due to the risk of a vaccine induced increase of natural HZ cases, as predicted by several mathematical models. In reference [16], we have shown, by means of numerical optimization, that the adoption of time-varying vaccination programs can optimally manage the perverse effects of varicella vaccine on natural HZ. However, a critical issue that needs to be carefully considered is the choice of an appropriate VZV model to be used in the optimization control problems. In this paper, we have evaluated the results obtained by two different mathematical models for the simulation of VZV dynamics: one more complex and realistic (ASM), the other one simpler and with a reduction of complexity, in terms of the size of the parameter space e (LRM). As expected, LRM shows a reduced computational burden, capturing, in a qualitative manner, the main response trends of the ASM to the optimization problem.

The first interesting result is that by increasing the relative HZ cost, both models suggest the administration of reduced levels of vaccination and this remains valid in the case of both the full optimal control problem and the logistic one. Another interesting result is that, considering the free optimization with the augmented cost functional, optimal vaccination programs are qualitatively comparable. Overall, by using RLM, we have been able to reproduce all the qualitative findings of reference [16], therefore largely confirming the qualitative robustness of ASM results.

However, under different aspect, RLM appears as oversimplified due to the lack of age-heterogeneities in the main involved processes of varicella dynamics, generating unreliable predictions in regards of model time scales about herpes zoster. In particular, the epoch of the natural zoster boom predicted by LRM (under constant varicella vaccine uptake) lasts about 20 years, in disagreement with findings from the modeling literature [6, 8, 11] and ASM [16]. In summary, RLM is able to generate the main desired effects (namely the boom in natural herpes zoster and the slow increase of vaccine-related zoster following childhood varicella immunization) and on the other hand could be sufficiently simple in terms of parameter definition in order to concretely assist public health decisions on infection and vaccination, also in countries where a complete dataset for a more complex parameterization is not available.

\section{Glossary of terms}

CMI Cell-mediated immunity.

HZ Herpes Zoster

VZV Varicella-Zoster Virus

\section{Appendix 1 Effects of considering different updating times of the control function}

We report here and compare the effects of three different values of the updating times of the control function, namely 1 year, which is the baseline level adopted in the main text, vs 6 and 3 months, respectively, on the optimal temporal trend of the varicella vaccination function for the LRM model. The comparison reported below has been carried out for different levels of the relative cost of herpes zoster $\left(C_{z}=1, C_{z}=5, C_{z}=20\right)$, over a time horizon of 50 years. 


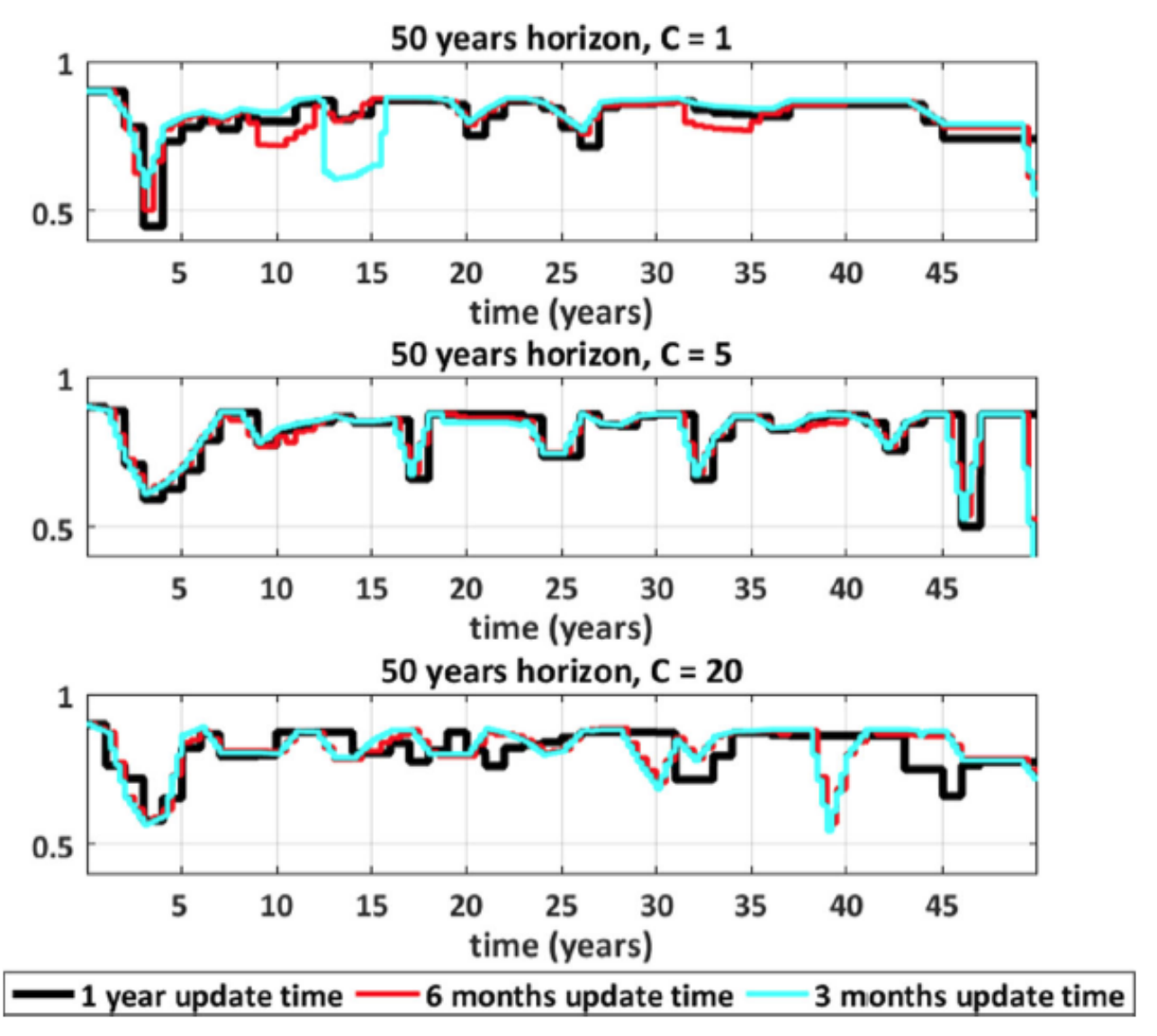

Fig. 6 The LRM model. A comparison of the temporal trends of the optimal control function for distinct values of the updating time: 1 year (black line), which is the benchmark considered in the main text, versus 6 months and 3 months, respectively, in red and cyano. The comparison is made for different levels of herpes zoster disease $(\mathrm{Cz}=1, \mathrm{Cz}=5, \mathrm{Cz}$ = 20).

\section{References}

1. Heymann DL (2004) Control of communicable diseases, 18th edn. American Public Health Association, Washington

2. Hope-Simpson RE (1965) The nature of herpes zoster: a long-term study and a new hypothesis. Proc R Soc Med 58:9-20

3. Oxman MN (2009) Herpes Zoster pathogenesis and cell-mediated immunity and immunosenescence. J Am Osteopath Assoc 109(6 Suppl 2):S13-7

4. Stefanoff P, Polkowska A, D’Ancona FP, Giambi C, Bruhl DL, O’Flanagna D, Dematte L, Lopalco P, and Johansen K (2010)

Varicella and herpes zoster surveillance and vaccination reccommendations 2010-2011, ${ }^{\wedge}$ Vaccine Eropean New Integrated

Collaboration Effort. VENICE II CONSORTIUM

5. Schuette M (1999) Modeling the effects of varicella vaccination programs on the incidence of chickenpox and shingles. Bull Math Biol 61(6):1031-1064

6. Brisson M, Edmunds WJ, Gay NJ, Law B, De Serres G (2000) Modelling the impact of immunization on the epidemiology of varicella zoster virus. Epidemiol Infect 125(3):651-669

7. van Hoek AJ, Melegaro A, Gay N, Bilcke J, Edmunds WJ (2012) The cost-effectiveness of varicella and combined varicella and herpes zoster vaccination programmes in the United Kingdom. Vaccine 30(6):1225-1234

8. Brisson M, Gay NJ, EdmundsWJ, Andrews NJ (2002) Exposure to varicella boosts immunity to herpes-zoster: implications for mass vaccination against chickenpox. Vaccine 20(19-20):2500-2507

9. Karhunen M, Leino T, Salo H, Davidkin I, Kilpi T, Auranen K (2010) Modelling the impact of varicella vaccination on varicella and zoster. Epidemiol Infect 138(4):469-481

10. Hales CM, Harpaz R, Joesoef MR, Bialek SR (2013) Examination of links between herpes zoster incidence and childhood varicella vaccination. Ann Intern Med 159(11):739

11. van Hoek AJ,Melegaro A, Zagheni E, EdmundsWJ, Gay N (2011) Modelling the impact of a combined varicella and zoster vaccination programme on the epidemiology of varicella zoster virus in England. Vaccine 29(13):2411-2420

12. Guzzetta G, Poletti P, Del Fava E, Ajelli M, Scalia Tomba GP, Merler S, Manfredi P (2013) Hope-Simpson's progressive immunity hypothesis as a possible explanation for herpes zoster incidence data. Am J Epidemiol 177(10):1134-1142

13. Poletti P, Melegaro A,Ajelli M, Del Fava E,Guzzetta G, Faustini L, Scalia Tomba G, Lopalco P, Rizzo C, Merler S, Manfredi P (2013) Perspectives on the impact of varicella immunization on herpes zoster. A model-based evaluation from three European countries. PLoS One 8(4):e60732

14. Marchetti S, Guzzetta G, Flem E, Mirinaviciute G, Scalia Tomba G, Manfredi P (2018) Modeling the impact of combined vaccination programs against varicella and herpes zoster in Norway. Vaccine

36(8):1116-1125 
15. Melegaro A, Marziano V, Del Fava E, Poletti P, Tirani M, Rizzo C, Merler S (2018) The impact of demographic changes, exogenous boosting and new vaccination policies on varicella and herpes zoster in Italy: a modelling and cost-effectiveness study. BMCMed 16(1):117 16. Betta M, Laurino M, Pugliese A, Guzzetta G, Landi A, Manfredi P (1826) Perspectives on optimal control of varicella and herpes zoster by mass routine varicella vaccination. Proc Biol Sci 283:2016

17. Civen R, Chaves SS, Jumaan A, Wu H, Mascola L, Gargiullo P, Seward JF (2009) The incidence and clinical characteristics of herpes zoster among children and adolescents after implementation of varicella vaccination. Pediatr Infect Dis J 28(11):954-959

18. Lenhart S and Workman JT (2007) Optimal control applied to biological models

19. Sharomi O, Malik T (2017) Optimal control in epidemiology. Ann Oper Res 251(1-2):55-71

20. Althaus CL (2014) Estimating the reproduction number of Ebola virus (EBOV) during the 2014 outbreak in West Africa. PloS Curr

21. Lewnard JA, Ndeffo Mbah ML, Alfaro-Murillo JA, Altice FL, Bawo L, Nyenswah TG, Galvani AP (2014) Dynamics and control of Ebola virus transmission in Montserrado, Liberia: a mathematical modelling analysis. Lancet Infect Dis 14(12):1189-1195

22. van Hoek AJ, Gay N, Melegaro A, Opstelten W, Edmunds WJ (2009) Estimating the cost-effectiveness of vaccination against herpes zoster in England and Wales. Vaccine 27(9):1454-1467

23. Nardone A, de Ory F, CartonM, Cohen D, van Damme P, Davidkin I, Rota MC, de Melker H, Mossong J, Slacikova M, Tischer A, Andrews N, Berbers G, Gabutti G, Gay N, Jones L, Jokinen S,Kafatos G, de Aragón MVM, Schneider F, Smetana Z, Vargova B, Vranckx R, Miller E (2007) The comparative seroepidemiology of varicella zoster virus in 11 countries in the European region. Vaccine 25(45):78667872

24. Goeyvaerts N, Hens N, Ogunjimi B, Aerts M, Shkedy Z, Van Damme P, Beutels P (2010) Estimating infectious disease parameters from data on social contacts and serological status. J R Stat Soc Ser C (Appl Stat) 59(2):255-277

25. BrissonM(2003) Varicella vaccination in England andWales: costutility analysis. Arch Dis Child 88(10):862-869

26. Bilcke J, Ogunjimi B, Marais C, de Smet F, Callens M, Callaert K, van Kerschaver E, Ramet J, van Damme P, Beutels P (2012) The health and economic burden of chickenpox and herpes zoster in Belgium. Epidemiol Infect 140(11):2096-2109

27. Pontryagin LS (1987) Mathematical theory of optimal processes

28. The Mathworks Inc, Matlab. 2009 